\title{
Psychosocial interventions for the prevention of relapse in bipolar disorder: systematic review of controlled trials
}

\author{
Suzanne Beynon, Karla Soares-Weiser, Nerys Woolacott, Steven Duffy and John R. Geddes
}

\section{Background}

Pharmacological interventions alone do not provide sufficient benefit for some individuals with bipolar disorder.

\section{Aims \\ To determine the effectiveness of psychosocial interventions for the prevention of relapse in bipolar disorder \\ Method \\ A systematic review and meta-analysis of randomised or quasi-randomised controlled trials were conducted.}

\section{Results}

Cognitive-behavioural therapy or group psychoeducation may be effective for relapse prevention in stable individuals. Family therapy was no more or less effective than individual psychosocial therapy or crisis management. There is no evidence that care management or integrated group therapy is effective in the prevention of relapse.

\section{Conclusions}

Cognitive-behavioural therapy, group psychoeducation and possibly family therapy may be beneficial as adjuncts to pharmacological maintenance treatments.

\section{Declaration of interest}

J.G. has received research funding from GlaxoSmithkline, Sanofi-Aventis, the UK Department of Health and Medical Research Council, the Stanley Medical Research Institute, and advisory committee payments from Bristol Myers Squibb.
Bipolar disorder is a complex, recurrent mood disorder, and its impact on everyday life can be devastating. Although pharmacological interventions remain the primary tool in its management, medicines cannot control all aspects and consequences of the disorder. Psychosocial interventions target issues untouched by pharmacological treatments, such as medication adherence, awareness and understanding of the disorder, early identification of prodromal symptoms, and coping skills. When combined with long-term pharmacological treatment, psychosocial interventions may enable individuals to take a more active role in the management of their disorder, and lead to improvements in mood stability, occupational and social functioning, and quality of life. ${ }^{1-4}$

We conducted a systematic review to determine whether psychosocial interventions could be effective in reducing relapse in people with bipolar disorder. The review was commissioned by the UK National Institute for Health Research's Health Technology Assessment Programme, which has published a full report. $^{5}$

\section{Method}

\section{Inclusion criteria}

We reviewed randomised or quasi-randomised controlled clinical trials with a follow-up period of at least 3 months' duration that investigated the effectiveness of any psychosocial intervention used for the prevention of relapse in bipolar disorder. A psychosocial intervention was considered to be any non-pharmacological intervention that aimed to improve the psychological and social functioning of the patient, in either an individual or a group setting. To be included in this review, the intervention had to be used for the maintenance treatment of bipolar disorder - i.e. target the prevention of further episodes, after patients were already stabilised following an acute bipolar episode. Included studies had to assess individuals with either type I or type II bipolar disorder, or a combination of the two. Type I disorder is defined as the occurrence of one or more manic episodes, often accompanied by one or more major depressive episodes; type II disorder is defined as the occurrence of one or more major depressive episodes, accompanied by at least one episode of hypomania. ${ }^{6}$

\section{Outcomes}

The primary outcome measure was all relapses, defined either as the number of hospitalisations in each group, the number of patients who received additional treatment, or as stated by the primary study authors. Relapses were defined 'as stated by authors' only when the authors provided no definition of relapse, or when definitions other than hospital admission or institution of additional treatment were used, e.g. emergence of a new acute episode, often defined according to DSM-IV criteria, scores on depression or mania rating scales, or a combination of the two. Although the authors' own definitions of relapse may have varied between trials, the definitions were similar enough to permit meaningful comparisons when using a relative measure of effect, although caution should be used when comparing absolute rates. Secondary outcome measures were manic and depressive relapses (also defined either as the number of hospitalisations in each group, the number of patients who received additional treatment, or as stated by the authors); adverse events leading to discontinuation; other treatment-related adverse events; and suicide or suicide attempts.

\section{Search strategy}

The databases Medline (1966 to 2005), PreMedline (September 2005), EMBASE (1980 to 2005), CINAHL (1982 to 2005), BIOSIS (1985 to 2005), PsycINFO (1872 to 2005), the Science Citation Index (1900 to 2005), Latin American and Caribbean Health Sciences Literature (LILACS) (1982 to 2005) and the Cochrane Central Register of Controlled Trials (2005:3) were searched using appropriate terms. A methodological search filter was used to help identify randomised and quasi-randomised controlled trials. Information on studies in progress, unpublished research or research reported in the grey literature was sought by searching 
a range of other databases, including Inside Conferences (1990 to 2005), ISI Proceedings: Science and Technology (1993 to 2005), the National Research Register (2005:3) and the National Technical Information Service (1990 to 2005). The search was not restricted by language. In addition, internet searches were carried out and selected conference abstracts were searched by hand. Further details of the search strategy can be obtained from the authors.

The titles and abstracts of all papers identified by the search were screened, and the full paper manuscripts for all potentially relevant studies were obtained and screened according to prespecified criteria. Because the literature search was broad, covering all treatments for bipolar disorder, broad inclusion criteria were applied during study selection, with those specific to psychosocial interventions applied at the final stage (Fig. 1). All papers that did not meet the inclusion criteria were excluded and the decisions for exclusion documented. Disagreements over the inclusion decision were resolved by consensus, or with the decision of a third reviewer. Where there was insufficient information reported to make a decision, or insufficient data for the study to be included, study authors were contacted for further details; if details were not forthcoming the studies were excluded.

\section{Data extraction and quality assessment}

Data extraction and quality assessment were conducted by one reviewer and checked independently by a second reviewer. Disagreements were resolved by consensus or with the decision of a third reviewer. Data were extracted into a pre-defined Microsoft Access database. The methodological design of all included trials was assessed according to quality criteria adapted from those in the Centre for Reviews and Dissemination's guidance for undertaking systematic reviews. ${ }^{7}$

\section{Data analysis}

The data were analysed using Stata version 8.2 and StatsDirect version 2.4.1 for Windows. Dichotomous data were analysed by calculating the odds ratio and $95 \%$ confidence intervals for each trial. For studies that presented data for more than one period of follow-up, data from the longest follow-up time point were used. Where there was more than one study for a comparison, the odds ratios were pooled using a fixed effect model (the Mantel-Haenszel method) and the corresponding confidence intervals were calculated. ${ }^{8}$ Statistical heterogeneity was assessed using the chi-squared test and expressed as $I^{2}$. The $I^{2}$ statistic describes the proportion of total variance across trials that is due to heterogeneity rather than chance. ${ }^{9}$

For the main analysis, odds ratios were calculated using the number of patients analysed as the denominator. The potential impact of the missing data was explored using sensitivity analyses. ${ }^{10}$ Sensitivity analysis was used to test best-case and worstcase scenarios for the primary outcome (all relapses). For the best-case scenario, the number of patients randomised was used as the denominator (i.e. assuming all patients who had not been analysed had not had a relapse). For the worst-case scenario, the number of patients randomised was used as the denominator and the difference between the number analysed and number randomised was added to the numerator (i.e. assuming all patients who were not included in the analysis had relapsed).

Studies in which patients (although receiving maintenance therapy) were randomised during the acute phase of bipolar disorder were not included in the main analysis, but were included in secondary analyses of the primary outcome (all relapses) only. Where there was only a single study for a comparison, and that

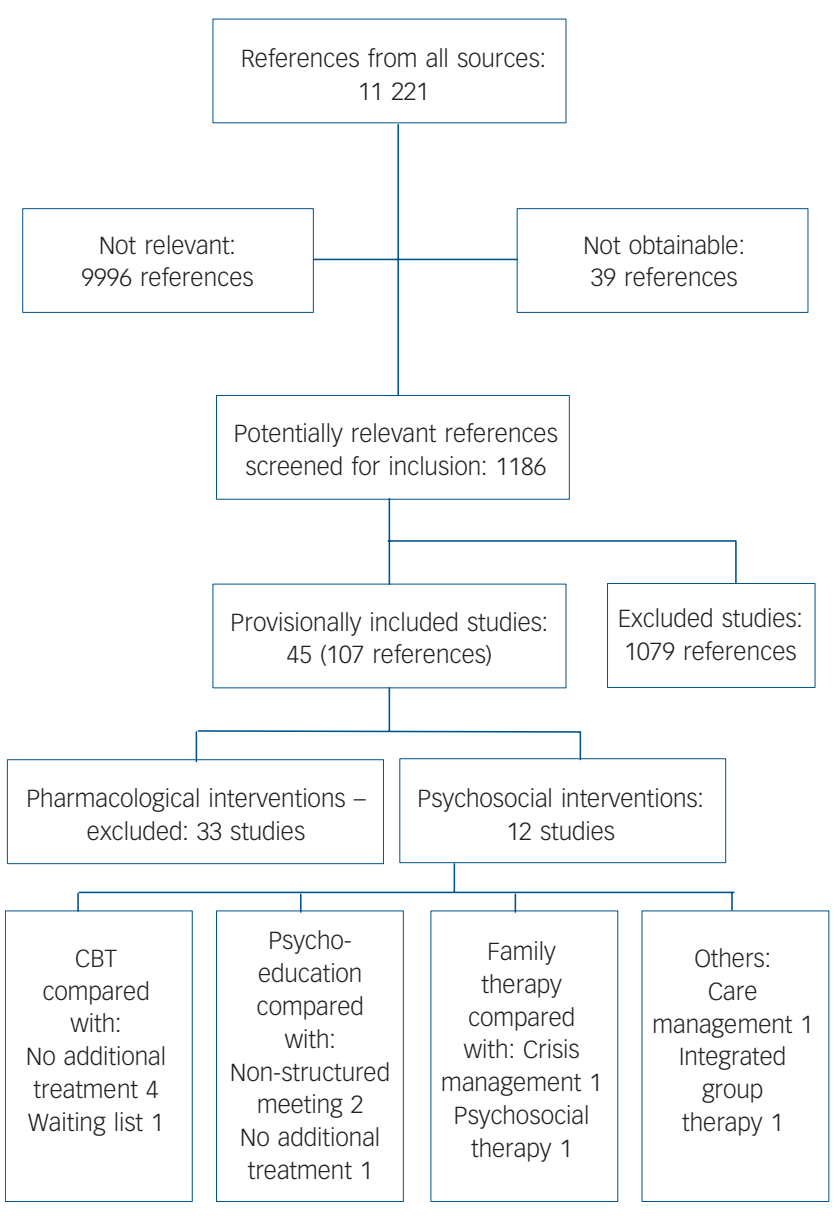

Fig. 1 Selection of included studies for the review.

$\mathrm{CBT}$, cognitive-behavioural therapy.

study was one that would have been included in a secondary analysis only, then results for that study were presented as for the main analysis. Where provided, data were analysed for manic and depressive relapses separately. For studies where mixed relapse was clearly defined as at least one manic episode (and additional depressive episode), the number of patients experiencing a mixed relapse was added to the number of patients with a manic relapse. Where available, data for suicide and adverse events were analysed for each comparison.

\section{Results}

\section{Included trials}

We identified 1225 potentially relevant references, 39 of which proved to be unobtainable. Twelve trials examined the effectiveness of psychosocial interventions and are included in this review (Fig. 1). The included studies evaluated cognitive-behavioural therapy (five studies), family therapy (two studies), psychoeducation (three studies), care management (one study) and integrated group therapy (one study) as adjuncts to usual pharmacological treatment. All studies were of patients with type I bipolar disorder or types I and II combined: none of the included studies presented data for patients with type II disorder alone. Details of the included studies and their methodological quality are presented in online Tables DS1 and DS2. Sample sizes and length of treatment and follow-up varied across studies. Although the overall methodological quality of some studies was sound, with all but 


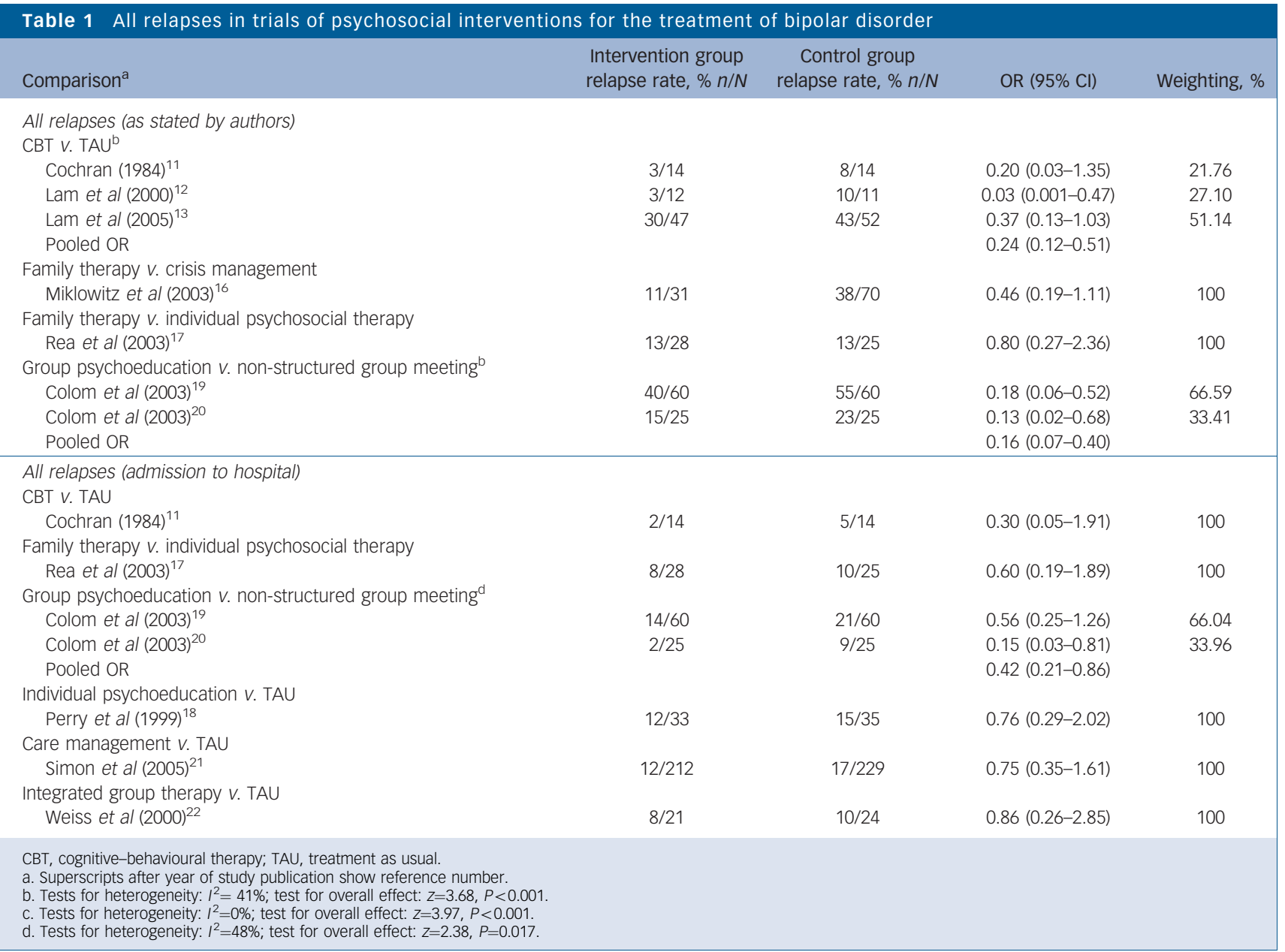

one reporting random allocation and some using assessor masking (blinding), several trials had small numbers of participants, providing limited data. In addition, poor reporting of methodological details - particularly in terms of method of randomisation, allocation concealment and masking of assessors - made full assessment of the quality of some studies difficult.

The results for all relapses and for manic and depressive relapses are presented in Tables 1 and 2 respectively. Where data were pooled across trials, unless otherwise stated, there was no evidence of substantial statistical heterogeneity. In all cases where a sensitivity analysis could be performed the findings did not differ from the main analysis. No information on suicide or adverse effects of the interventions was reported in any study.

\section{Cognitive-behavioural therapy}

Four studies compared cognitive-behavioural therapy (CBT) with treatment as usual (TAU) $)^{11-14}$ and one compared CBT with a waiting-list control. ${ }^{15}$ Two trials were excluded from the main analysis of the outcome 'all relapses ${ }^{14,15}$ because they randomised participants who were in an acute phase of bipolar disorder. One of these trials, ${ }^{14}$ which was of good quality and much larger than any of the other CBT trials, found no effect of CBT relative to TAU $(\mathrm{OR}=1.08,95 \%$ CI $0.66-1.77)$. The smaller trial ${ }^{15}$ also found no significant difference between $\mathrm{CBT}$ and a waiting-list control $(\mathrm{OR}=0.47,95 \% \mathrm{CI} 0.004-5.68)$. Of the remaining three trials, that by $\mathrm{Lam}^{13}$ had a larger sample size $(n=103)$, used masking of the outcome assessor and had a follow-up of 30 months. The results from this trial indicated a relative effect in favour of CBT but it was not statistically significant $(O R=0.37,95 \%$ CI $0.13-1.03)$. When these three trials ${ }^{11-13}$ were combined, there was no statistical heterogeneity and the resultant pooled odds ratio was statistically significant in favour of CBT for all relapses as stated by authors ( $\mathrm{OR}=0.2495 \% \mathrm{CI} 0.12-0.51)$. When the two excluded trials were added to the analysis, the beneficial effect of CBT on all relapses as stated by authors was reduced, but remained statistically significant $(\mathrm{OR}=0.63,95 \% \mathrm{CI} 0.43-0.94)$. However, these studies introduced between-study differences, and significant statistical heterogeneity was detected $\left(I^{2}=72 \%\right)$. The one study that provided data for relapse defined as admission to hospital found no significant difference between treatment groups $(\mathrm{OR}=0.30,95 \%$ CI $0.05-1.91)$.

One reasonably good-quality $\operatorname{trial}^{13}(n=103$, with 30 months follow-up) provided data for manic and depressive relapses, comparing CBT with TAU. Although this study found no statistically significant difference between treatment groups for the prevention of manic relapses ( $\mathrm{OR}=0.48,95 \% \mathrm{CI} 0.21-1.13)$, there were significantly fewer depressive relapses in the CBT group than in the TAU group $(\mathrm{OR}=0.32,95 \%$ CI $0.13-0.74)$.

\section{Family therapy}

Two studies investigated family therapy. ${ }^{16,17}$ One, a small study with some limitations in quality $(n=53)$, found no statistically significant difference between family therapy and individual psychosocial therapy for relapse defined as admission to hospital 
Manic relapses (as stated by authors)

CBT v. TAU

Lam et al $(2005)^{13}$

Family therapy $v$. crisis management Miklowitz et al (2003) ${ }^{16}$

Group psychoeducation v. non-structured group meeting ${ }^{\mathrm{b}}$ Colom et al (2003) ${ }^{19}$

Colom et al $(2003)^{20}$

$23 / 46$

$5 / 31$

$31 / 46$

$0.48(0.21-1.13)$

Pooled OR

$28 / 60$

$12 / 25$

$12 / 70$

$0.93(0.31-2.82)$

Manic relapses (admission to hospital)

Individual psychoeducation $v$. TAU

Perry et al (1999) ${ }^{18}$

Care management $v$. TAU

Simon et al (2005) ${ }^{21}$

Depressive relapses (as stated by authors)

CBT v. TAU

Lam et al $(2005)^{13}$

Family therapy $v$. crisis management Miklowitz et al (2003) ${ }^{16}$

Group psychoeducation $v$. non-structured group meeting ${ }^{c}$ Colom et al $(2003)^{19}$

Colom et al $(2003)^{20}$

Pooled OR

$0.27(0.14-0.53)$

Depressive relapses (admission to hospital)

Individual psychoeducation $v$. TAU Perry et al (1999) ${ }^{18}$

Care management $v$. TAU Simon et al $(2005)^{21}$

$39 / 169$

$58 / 182$

$0.64(0.40-1.03)$

BT, cognitive-behavioural therapy; TAU, treatment as usual.

a. Superscripts after year of study publication show reference numbers.

b. Test for heterogeneity: $I^{2}=0 \%$; test for overall effect: $z=3.86, P<0.001$

c. Test for heterogeneity: $1^{2}=0 \%$; test for overall effect: $z=4.37, P<0.001$

$(\mathrm{OR}=0.6,95 \%$ CI $0.19-1.89)$ or as stated by the authors $(\mathrm{OR}=0.80,95 \%$ CI $0.27-2.36) .{ }^{17}$ The second study, which was larger and of better quality, ${ }^{16}$ found that family therapy was not statistically significantly better than crisis management for relapse as stated by the authors $(\mathrm{OR}=0.46,95 \%$ CI $0.19-1.11)$, nor for prevention of manic or depressive relapse as stated by the authors (manic relapse, $\mathrm{OR}=0.93$, 95\% CI 0.31-2.82; depressive relapse, $\mathrm{OR}=0.41,95 \% \mathrm{CI} 0.15-1.12$ ). The failure to detect any treatment difference may be due to the small sample sizes. Furthermore, the control treatments used in both these trials of family therapy were to some extent active therapies, and therefore the results indicate that family therapy might have some beneficial effect; further investigation is warranted.

\section{Psychoeducation}

A total of three randomised trials that investigated the efficacy of psychoeducation were identified for the review. ${ }^{18-20}$ Two trials of reasonable quality, both with 24 months of follow-up, and the larger of which used assessor masking, investigated group psychoeducation in comparison with unstructured group meetings. ${ }^{19,20}$ The pooled odds ratios were statistically significant in favour of group psychoeducation for all relapses defined as admission to hospital $(\mathrm{OR}=0.42,95 \% \mathrm{CI} 0.21-0.86)$ and as stated by the authors $(\mathrm{OR}=0.16,95 \% \mathrm{CI} 0.07-0.40)$, and there were significantly fewer manic and depressive relapses in participants attending group psychoeducation than in participants attending non-structured group meetings (manic relapse, $\mathrm{OR}=0.27,95 \%$ CI 0.14-0.53; depressive relapse, $\mathrm{OR}=0.24,95 \%$ CI $0.12-0.45$ ).
The third trial, which also used assessor masking, but did not provide adequate details of methods of allocation concealment, compared individual psychoeducation with TAU. ${ }^{18-20}$ The results show no significant difference between groups for the prevention of all relapses defined as admission to hospital ( $\mathrm{OR}=0.76,95 \% \mathrm{CI}$ 0.29-2.02). There were significantly fewer manic relapses, defined as admission to hospital, in participants receiving psychoeducation than in participants receiving TAU $(\mathrm{OR}=0.28,95 \% \mathrm{CI}$ $0.10-0.78)$. However, there was no statistically significant difference in the rate of depressive relapses, defined as admission to hospital, between the treatment groups $(\mathrm{OR}=2.03,95 \% \mathrm{CI}$ 0.77-5.35).

\section{Care management}

One good-quality, assessor-masked randomised trial with a large sample size $(n=441)$ and 12 months follow-up investigated the efficacy of care management compared with TAU. ${ }^{21}$ The results for all relapses showed no significant difference between care management and TAU for all relapse, defined as admission to hospital $(\mathrm{OR}=0.75,95 \%$ CI $0.35-1.61)$. Although the study was of good quality, it should be noted that this result included participants who entered the study while still in an acute phase of bipolar disorder, which might have influenced relapse rates. There was no statistically significant difference between care management and TAU for manic or depressive relapses defined as admission to hospital (manic relapse, $\mathrm{OR}=0.64,95 \%$ CI $0.40-1.03$; depressive relapse, $\mathrm{OR}=1.00,95 \%$ CI $0.62-1.62$ ). 


\section{Integrated group therapy}

One small, poor-quality quasi-randomised trial with no masking that investigated the efficacy of integrated group therapy compared with $\mathrm{TAU}^{22}$ found no statistically significant difference between the treatments for all relapses defined as admission to hospital $(\mathrm{OR}=0.86,95 \% \mathrm{CI} 0.26-2.85)$. The trial did not provide any data for manic or depressive relapses separately. The study reported one suicide attempt in the TAU group and none in the integrated therapy group.

\section{Discussion}

We used rigorous systematic methods to review all randomised and quasi-randomised trials of psychosocial treatments for the prevention of relapse in bipolar disorder. Our comprehensive literature searches and use of fairly broad inclusion criteria should have ensured we were unlikely to have missed any relevant studies. Only a small number of randomised controlled trials were identified for our review, and there were not many repetitions of specific comparisons. Thus few of the treatments were thoroughly investigated. Furthermore, although we restricted inclusion to randomised and quasi-randomised controlled trials, the methodological quality of some of these trials was limited, and the strength of evidence was not equal for all treatments or for all comparisons.

The outcomes that could be summarised were also limited. Relapse rates were reported differently in the various trials, and some trials reported data separately for manic or depressive relapses whereas others reported all relapses only. The duration of follow-up varied across the trials. However, all used a minimum period of 6 months, and all interventions except integrated group therapy were tested under follow-up of 12 months or more. Our compromise in using the data only from the longest available follow-up for each trial is likely to have introduced some degree of inaccuracy and to have masked or exaggerated some treatment effects or differences.

We had hoped to look at the effects of interventions on suicide rates, but unfortunately data on suicide were extremely scarce. Similarly, no data on adverse effects were reported. Further research examining potential adverse effects of psychosocial interventions is required, so that decisions regarding the efficacy and use of psychosocial interventions can be made in light of possible adverse events. ${ }^{23}$

A potential problem in some of these results may be that of publication bias, whereby studies with statistically significant results are more likely to be published than those with nonsignificant results. Given the small numbers of studies for each outcome, formal assessment of publication bias was not possible; however, the potential for overestimation of treatment effects cannot be discounted. That said, we made considerable efforts to identify unpublished studies in our literature searches, and more than half of the comparisons made were based on single-study results that found no significant treatment effect.

Another potential problem arose from the use of 'treatment as usual' control groups in the primary studies. Seven of the studies included in this review described the control as 'treatment as usual' or 'no additional treatment', and yet no two control groups received the same intervention. What is considered to be usual treatment may vary from one clinical setting to another, and certainly from one country to another, with differences in drug licensing and treatment guidelines. In addition, the studies included here span over 20 years, and what was considered usual treatment for bipolar disorder in the 1980s may be very different from standard treatment today. This not only has implications in terms of whether it is appropriate to pool clinically heterogeneous studies, but may also affect how estimates of treatment effects can be generalised to current clinical practice.

\section{Clinical findings}

In general, the studies investigating psychosocial interventions were small, and there were few data for each comparison and outcome, making it difficult to draw any firm conclusions. The available evidence did suggest that cognitive-behavioural therapy, in combination with usual treatment, is effective for the prevention of relapse in patients with stable disorders. However, some of the studies included in the pooled analysis were of limited quality. There is reasonably good evidence that group psychoeducation is more effective than non-structured meetings for preventing all relapses, manic relapses and depressive relapses. In contrast, evidence from a single small trial found that although individual psychoeducation was more effective than TAU for the prevention of manic relapses, it was no more effective than TAU for prevention of all relapses or depressive relapses. Given that non-structured meetings are a more active control than TAU, one would expect, if anything, a larger treatment effect in the comparison with TAU, but this was not the case. Reasons for this are unclear; however, the poorer quality and small sample size of the study comparing psychoeducation with TAU may be contributing factors. Alternatively, differences in 'dosage' and duration of psychoeducation between studies or - perhaps more importantly - the individual $v$. group context in which the interventions were delivered might have been responsible.

Neither of the trials of family therapy found a significant treatment effect; however, the control treatments used in both these trials were to some extent active therapies and therefore the possibility that family therapy might have some beneficial effect cannot be discounted; further research is required. There was no evidence that care management or integrated group therapy is effective in the prevention of relapse, but this reflects a lack of evidence rather than any evidence of a lack of effect.

Unfortunately, the two best-quality trials randomised patients to treatment groups while they were experiencing an acute episode, and therefore do not provide direct information about prevention of relapse in the euthymic stage only. Interestingly, both the trial of $\mathrm{CBT}^{14}$ and the trial of care management ${ }^{21}$ found that these psychosocial interventions did not provide additional benefit to standard pharmacological therapy when used for the treatment of an acute episode and maintenance therapy. This may suggest that CBT or care management or other psychosocial interventions are only effective in stable patients. However, this evidence is by no means conclusive. A recent randomised controlled trial, restricted to patients with type I or type II bipolar disorder experiencing a current major depressive episode, found intensive psychotherapy (CBT, family-focused therapy or interpersonal social rhythm therapy) to be more effective than a brief psychoeducational intervention in reducing time to recovery and increasing the likelihood that patients would be well in any study month. ${ }^{24}$

The suggestion that, in general, psychosocial interventions as adjuncts to pharmacological treatment can be effective in individuals with bipolar disorder is in keeping with the results of previous systematic reviews, ${ }^{25-27}$ and evidence for CBT and psychoeducation in particular has been noted. ${ }^{3}$ However, it also should be noted that the majority of the available studies evaluating psychosocial interventions were carried out by researchers who are proponents of and experts in these interventions, and it is possible that treatment effects might not be as great when the interventions are applied and evaluated by others.

A recent review and meta-analysis of randomised controlled trials compared psychological therapies plus standard psychiatric 
care with standard psychiatric care alone, and found a statistically significant benefit of adjunctive psychological therapies in the prevention of relapse. ${ }^{27}$ Furthermore, it suggested that these interventions were most effective in preventing relapse in patients who were euthymic. Because our review was not limited to randomised controlled trials of these comparators, we were able to include three additional studies that were not included in the metaanalysis of Scott and colleagues. ${ }^{17,21,22}$ One additional study included in the Scott review was a randomised controlled trial of interpersonal social rhythm therapy. ${ }^{28}$ This study provided relapse data for both acute and stable phases of bipolar disorder combined, and therefore could not be included in our review. Whereas in general the results of the meta-analysis supported the findings of our review, the studies pooled by Scott et al ${ }^{27}$ differed from one another considerably, in terms of both interventions and populations. Consequently, it is not clear how meaningful their pooled results are, or to which interventions or populations the results can be generalised. Furthermore, the statistical analysis did not take into account the fact that results for each intervention type came from different studies; neither did the authors take into account the quality of studies included in the analysis.

Overall, our review found no evidence from which to draw conclusions regarding the relative efficacy of the different psychosocial interventions. Insufficient data and a lack of common comparators meant that an indirect meta-analysis to compare the different interventions across trials was not possible. This finding reflects previous reviews which have also found little evidence to recommend one type of psychosocial intervention above another. ${ }^{25,29}$ There is probably some overlap in the different components of these interventions, and even though some have different theoretical bases, they have targets and strategies in common. ${ }^{23,25,27,30}$ For example, many of the interventions aimed to promote awareness and understanding of the disorder, increase medication adherence and to improve early identification of prodromal symptoms. ${ }^{27}$ Future research might therefore be best directed at identifying which components of these interventions are most effective rather than comparing the different intervention packages as a whole.

The task of identifying the 'active' components of an intervention can be tackled in both primary and secondary research. Careful documentation and a staged approach to the development and assessment of psychosocial interventions in primary studies, incorporating both qualitative and quantitative methods, can aid in the evaluation of complex interventions such as these. ${ }^{31}$ In secondary research, techniques such as meta-regression have been used to determine which aspects of an intervention may predict treatment effects. In a recent systematic review of 37 randomised controlled trials evaluating collaborative care for the treatment of depression, Gilbody and colleagues ${ }^{32}$ used meta-regression in order to try to identify possible 'active' components of the intervention. Although our review did not comprise a sufficient number of studies to perform meta-regression in this way, this approach could be used in the future as further better-quality research arises. This approach might also help to elucidate how the beneficial effects of psychosocial interventions on relapse rates come about, and whether psychosocial interventions can directly help to prevent relapse, or whether they serve only to enhance the effects of pharmacological treatment. The results of the meta-regression conducted by Gilbody and colleagues showed that collaborative care significantly improved adherence to medication, and found a dose-response relationship between medication use and improvements in depression. It is possible that the same would be true in the treatment of bipolar disorder; however, at present the relationship between pharmacological and psychosocial interventions in this setting remains unclear.

\section{Future research}

There is growing interest in the development of psychosocial interventions for patients with bipolar disorder but these have not yet been investigated thoroughly. There is some evidence that cognitive-behavioural therapy, group psychoeducation and possibly family therapy may be beneficial as adjuncts to pharmacological maintenance treatments for the prevention of relapse in stable patients. Well-conducted trials of all psychosocial interventions as adjuncts to pharmacological maintenance treatments are required. Such trials would be properly randomised and powered, the assessor masked, and ideally would use a standardised control. A more detailed analysis of the different components of the psychosocial interventions would be helpful in determining which aspects of the interventions are most effective, by which indirect routes they might have their effect, and for whom they are most effective.

\footnotetext{
Suzanne Beynon, MSC, Karla Soares-Weiser, MD, PhD, Nerys Woolacott, BSC, PhD, Steven Duffy, BA(Hons), PgDip, Centre for Reviews and Dissemination, University of York, York, UK; John R. Geddes, MD, FRCPsych, Department of Psychiatry, University of Oxford, Oxford, UK

Correspondence: Nerys Woolacott, Centre for Reviews and Dissemination, University of York, York Y010 5DD, UK. Email: nw11@york.ac.uk

First received 12 March 2007, final revision 21 August 2007, accepted 6 September 2007
}

\section{Acknowledgements}

The authors thank Rob Riemsma for his considerable contribution in the initial stages of the review, James Coates for his hard work in creating and managing our study database, and Rebeca Trowman for her assistance with the data analysis. This project was funded by the UK Health Technology Assessment Programme (project number 05/35/01). The views and opinions expressed herein are those of the authors and do not necessarily reflect those of the UK Government Department of Health.

\section{References}

1 Scott J. Cognitive therapy as an adjunct to medication in bipolar disorder. $\mathrm{Br}$ J Psychiatry 2001; 178 (suppl. 41): s164-8.

2 Ghaemi SN, Pardo TB, Hsu DJ. Strategies for preventing the recurrence of bipolar disorder. J Clin Psychiatry 2004; 65 (suppl. 10): 16-23.

3 Vieta $\mathrm{E}$, Colom F. Psychological interventions in bipolar disorder: from wishful thinking to an evidence-based approach. Acta Psychiatr Scand Suppl 2004; 110: $34-8$

4 Vieta E. The package of care for patients with bipolar depression. J Clin Psychiatry 2005; 66 (suppl. 5): 34-9.

5 Soares-Weiser K, Bravo Vergel Y, Beynon S, Dunn G, Barbieri M, Duffy S, Geddes J, Gilbody S, Palmer S, Woolacott N. A systematic review and economic model of the clinical effectiveness and cost effectiveness of interventions for preventing relapse in people with bipolar disorder. Health Technol Assess 2007; 11(39).

6 American Psychiatric Association. Diagnostic and Statistical Manual of Mental Disorders (4th edn) (DSM-IV). American Psychiatric Association, 2000

7 NHS Centre for Reviews and Dissemination. Undertaking Systematic Reviews of Research on Effectiveness: CRD's Guidance for Carrying out or Commissioning Reviews. CRD Report 4 (2nd edn). University of York, 2001.

8 Higgins JPT, Green S (ed). Cochrane Handbook for Systematic Reviews of Interventions 4.2.5. Cochrane Library, issue 3. Wiley Interscience, 2005

9 Higgins JPT, Thompson SG, Deeks JJ, Altman DG. Measuring inconsistency in meta-analyses. BMJ 2003; 327: 557-60.

10 Deeks JJ, Higgins JPT, Altman DG. Analysing and presenting results. In Cochrane Handbook for Systematic Reviews of Interventions 4.2.6. (eds JPT Higgins, S Green), section 8, Cochrane Library, issue 4. Wiley Interscience 2006.

11 Cochran SD. Preventing medical noncompliance in the outpatient treatment of bipolar affective disorders. J Consult Clin Psychol 1984; 52: 873-8.

12 Lam DH, Bright J, Jones S, Hayward P, Schuck N, Chisham D, Sham P. Cognitive therapy for bipolar illness: a pilot study of relapse prevention. Cognit Ther Res 2000; 24: 503-20. 
13 Lam DH, Hayward P, Watkins ER, Wright K, Sham P. Relapse prevention in patients with bipolar disorder: cognitive therapy outcome after 2 years. Am J Psychiatry 2005; 162: 324-9.

14 Scott J, Paykel E, Morriss R, Bentall R, Kinderman P, Johnson T, Abbott R, Hayhurst H. Cognitive-behavioural therapy for severe and recurrent bipolar disorders. Randomised controlled trial. Br J Psychiatry 2006; 188: 313-20.

15 Scott J, Garland A, Moorhead S. A pilot study of cognitive therapy in bipolar disorders. Psychol Med 2001; 31: 459-67.

16 Miklowitz DJ, George EL, Richards JA, Simoneau TL, Suddath RL. A randomized study of family-focused psychoeducation and pharmacotherapy in the outpatient management of bipolar disorder. Arch Gen Psychiatry 2003; 60: 904-12.

17 Rea MM, Tompson MC, Miklowitz DJ, Goldstein MJ, Hwang S, Mintz J. Familyfocused treatment versus individual treatment for bipolar disorder: results of a randomized clinical trial. J Consult Clin Psychol 2003; 71: 482-92.

18 Perry A, Tarrier N, Morriss R, McCarthy E, Limb K. Randomised controlled trial of efficacy of teaching patients with bipolar disorder to identify early symptoms of relapse and obtain treatment. BMJ 1999; 318: 149-53.

19 Colom F, Vieta E, Martinez-Aran A, Reinares M, Goikolea HM, Benabarre A, Torrent C, Comes M, Corbella B, Parramon G, Corominas J. A randomized trial on the efficacy of group psychoeducation in the prophylaxis of recurrences in bipolar patients whose disease is in remission. Arch Gen Psychiatry 2003; 60: 402-7.

20 Colom F, Vieta E, Reinares M, Martínez-Arán A, Torrent C, Goikolea JM, Gastó C. Psychoeducation efficacy in bipolar disorders: beyond compliance enhancement. J Clin Psychiatry 2003; 64: 1101-5.

21 Simon GE, Ludman EJ, Unutzer J, Bauer MS, Operskalski B, Rutter C. Randomized trial of a population-based care program for people with bipolar disorder. Psychol Med 2005; 35: 13-24.

22 Weiss RD, Griffin ML, Greenfield SF, Najavits LM, Wyner D, Soto JA, Hennen JA. Group therapy for patients with bipolar disorder and substance dependence: results of a pilot study. J Clin Psychiatry 2000; 61: 361-7.
23 Gonzalez-Pinto A, Gonzalez C, Enjuto S, de Corres BF, Lopez P, Palomo J, Gutierrez M, Mosquera F, de Heredica JL. Psychoeducation and cognitivebehavioral therapy in bipolar disorder: an update. Acta Psychiatr Scand 2004; 109: 83-90

24 Miklowitz DJ, Otto MW, Frank E, Reilly-Harrington NA, Wisniewski SR, Kogan $J N$, Nierenberg AA, Calabrese JR, Marangell LB, Gyulai L, Araga M, Gonzales JM, Shirley ER, Thase ME, Sachs GS. Psychosocial treatments for bipolar depression: a 1-year randomized trial from the Systematic Treatment Enhancement Program. Arch Gen Psychiatry 2007; 64: 419-26.

25 Scott J, Gutierrez MJ. The current status of psychological treatments in bipolar disorders: a systematic review of relapse prevention. Bipolar Disord 2004; 6: 498-503.

26 Rouget BW, Aubry JM. Efficacy of psychoeducational approaches on bipolar disorders: a review of the literature. J Affect Disord 2007; 98: 11-27.

27 Scott J, Colom F, Vieta E. A meta-analysis of relapse rates with adjunctive psychological therapies compared to usual psychiatric treatment for bipolar disorders. Int J Neuropsychopharmacol 2007; 10: 123-9.

28 Frank E, Kupfer DJ, Thase ME, Mallinger AG, Swartz HA, Fagiolini AM, Grochocinski V, Houck P, Scott J, Thompson W, Monk T. Two-year outcomes for interpersonal and social rhythm therapy in individuals with bipolar I disorder. Arch Gen Psychiatry 2005; 62: 996-1004.

29 Scott J. Psychotherapy for bipolar disorders: efficacy and effectiveness. J Psychopharmacol 2006; 20: 46-50.

30 Miklowitz DJ. A review of evidence-based psychosocial interventions for bipolar disorder. J Clin Psychiatry 2006; 67: 28-33.

31 Campbell M, Fitzpatrick R, Haines A Kinmonth AL, Sandercock $P$, Spiegelhalter $D$, Tyrer P. Framework for design and evaluation of complex interventions to improve health. BMJ 2000; 321: 694-6.

32 Gilbody S, Bower P, Fletcher J, Richards D, Sutton AJ. Collaborative care for depression: a cumulative meta-analysis and review of longer-term outcomes. Arch Intern Med 2006; 166: 2314-21.

AP EXTRA CONTENT
ONLINE

\section{Word pictures of depression: low mood}

\section{Sharon Mcconville}

When a doctor asks whether a patient is 'low in spirits' or 'low in mood', what does he mean? For me, it was a very visceral feeling. I compared it to feeling like an empty shell or a dark cave.

'I feel like I am always going to have this hollow emptiness inside me. I feel like I am a shell within which there is infinite darkness, like a cave of which one can see only the mouth but which has a dark, slimy-walled interior extending deep into the cold bowels of the earth. It is musty in there and untold horrors lurk in its deepest recesses. The stagnant, icy water which lies upon the floor has been in that same state for years, removed from the cycle of transpiration, evaporation and condensation by its separation from the outside reality. There are parts of me which are just like this water: bitter and unpalatable, discoloured, stale. I used to be frightened by caves but intrigued at the same time by the possibility that treasures might lie within. I used to be frightened by the deepest invaginations of my soul but intrigued by the possibility that to explore them might yield great riches. Now the intrigue has faded: I expect to find not jewels and gold but black rock, threatening-looking stalactites and glutinous sludge. Thinking about my soul leaves me cold rather than excited. Nothing could be heartwarming enough to heat me to the core. Where is my heart, this part of me which has ached and has been broken and now is as numb with cold as if it had been preserved in liquid nitrogen? Will it ever thaw? One thing is certain: if it does, it will be even more painful than warming frost-bitten fingers on a winter's day.' 